\title{
A PROSPECTIVE STUDY ON THE ISOLATION, CULTURE, SUB-CULTURE AND SPECIES IDENTIFICATION OF CANDIDA FROM THE MUCOSAL LESIONS OF IMMUNO-COMPROMISED INDIVIDUALS
}

\author{
Rajkumar Kannan'1, Janaki Chellam², Kannan Ponnusamy³ \\ ${ }^{1}$ Assistant Professor, Department of Dermatology, Madras Medical College (MMC), Chennai, Tamilnadu. \\ 2Professor (Mycology), Department of Dermatology, Madras Medical College (MMC), Chennai, Tamilnadu. \\ ${ }_{3}^{3}$ Research Scholar, Department of Microbiology, Madras Medical College (MMC), Chennai, Tamilnadu.
}

\begin{abstract}
BACKGROUND

History of candidiasis dates back to $4^{\text {th }}$ century BC, when Hippocrates described oral aphthae (Thrush) in two patients with severe underlying disease. The initial discovery of thrush organism was made in 1839 by Langenbeck, who observed a fungus in the scrapings of buccal thrush from a patient with typhus. In 1849, Wilkinson first described vaginal candidiasis and its mycotic origin; the dimorphic nature of thrush fungi was noticed by Grawitz in 1877. Fungal infection in human beings ranges from those involving only the most superficial areas of the body and those in which subcutaneous tissue is involved and which sometimes can cause deep and systemic infections that often involved most of the major organs of the body, particularly in an immunocompromised background. Immuno-compromised individuals are those who have a compromised defence system of the body either directly as a result of the disease per se or as a result of treatment with immunosuppressive drugs that the primary ailment they suffer from warrants.

This study focuses mainly on the isolation, culture, sub-culture and species identification of candida from the mucosal lesions of immuno-compromised individuals.
\end{abstract}

\section{MATERIALS AND METHODS}

This descriptive study was conducted at the Government General Hospital, Chennai. One hundred and twenty (120) patients who were either on immunosuppressive therapy or suffering from acquired immunodeficiency states, attending the Department of Dermatology were screened for the present study. Cases were included in the study after application of inclusion and exclusion criteria and after confirming the diagnosis through appropriate investigations including histopathological and serological studies.

\section{RESULTS}

Out of sample size of 120 patients, 100 patients (males- 62, females- 38) were found to be positive for mucosal candidiasis. Following culture in Sabouraud's Dextrose Agar and sub-culture in the indicator media of HiCrome Candida Agar the following species of candida were isolated, viz. Candida albicans (52\%), C. tropicalis (22\%), C. glabrata (21\%), C. krusei (3\%) and C.dubliniensis (2\%).

\section{CONCLUSION}

Candidiasis is the commonest mycosis in patients on systemic immunosuppressive therapy and immunosuppressive status like HIV infection. Candida albicans was the commonest species isolated (52\%). Acute pseudomembranous glossitis was the commonest morphological type of lesion encountered in this study (35\%). Even in HIV infected individuals, Candida albicans was the commonest species isolated in our study.

\section{KEYWORDS}

Candida Species Identification, Indicator Media, Hi-Media Crome Agar, Candida Albicans.

HOW TO CITE THIS ARTICLE: Kannan R, Chellam J, Ponnusamy K. A prospective study on the isolation, culture, sub-culture and species identification of candida from the mucosal lesions of immuno-compromised individuals. J. Evolution Med. Dent. Sci. 2018;7(14):1741-1748, DOI: 10.14260/jemds/2018/393

\section{BACKGROUND}

Infections caused by the yeasts of the genus candida are common in clinical practice. They may involve the mucous membranes and/ or skin or spread internally to produce systemic infections. (1)

'Financial or Other Competing Interest': None.

Submission 12-03-2018, Peer Review 23-03-2018,

Acceptance 26-03-2018, Published 02-04-2018.

Corresponding Author:

Dr. Rajkumar Kannan,

Flat-C, New No. 3, Athipoo Flats,

Thiruvalluvar Street, Methanagar,

Chennai-600029, Tamilnadu.

E-mail: rajderm0002@gmail.com

DOI: $10.14260 /$ jemds/2018/393

\section{(c) (1) $(9)$}

Superficial infections of the mucous membranes and skin are numerically most important, but more serious involvement of the internal organs as in septicaemia, endocarditis and meningitis can also occur.

Candida albicans is an oval yeast $2-6 \times 3-9 \mu \mathrm{m}$ in size, which can produce budding cells, pseudohyphae and true hyphae. The ability to simultaneously display several morphological forms is known as polymorphism. Although, hyphae are likely to be produced during the process of tissue invasion, yeasts without hyphae may also occur in invasive disease, particularly in infections caused by non-albicans candida species.(2)

Candida species are normal commensals of the human beings. They are commonly found throughout the gastrointestinal tract, female genital tract and anterior 
urethra.(3) They cause opportunistic infections in man. They have many functions inside our digestive tract and one of them is to recognise and destroy harmful bacteria.

There are more than 200 species(1) of candida, but the following are considered to be important pathogens for humans: Candida albicans, Candida tropicalis, Candida krusei, Candida guilliermondii, Candida glabrata (Now classified as Torulopsis glabrata) and Candida parapsilosis. Candida albicans is the most commonly isolated species and accounts for $90 \%$ of cases of invasive Candidiasis. Trichosporon species which resembles Candida krusei have recently been isolated from clinical specimens. Candida species are the fifth most common primary bloodstream invaders and the seventh most common pathogen to cause nosocomial infections.(1)

Candida species grow easily on Sabouraud's Dextrose Agar at $37^{\circ} \mathrm{C}$ at room temperature within $24-48 \mathrm{hrs}$. The colonies are white or cream coloured, smooth with a yeasty odour that are naturally present as a part of the normal commensal in the digestive system. A problem can occur when there is an abnormal overgrowth of the yeast, that is normally controlled by the "beneficial bacteria" in the intestines. When factors such as antibiotics, steroids and refined sugar are used in excess, since bacteria and specific nutrients in the gut are destroyed imbalanced yeast can overgrow. The immune system and therefore our body's resistance is then lowered and the yeasts begin to invade and colonise the tissues.(4) These yeasts release toxic chemicals into the blood that causes varying symptoms. These toxins attack the immune system, permitting the fungi to continue their tissue invasion and to cause more serious symptoms.

The term immuno-compromised host is used to define a patient with impaired host defences who is at risk of developing an opportunistic infection. This includes patients with immunodeficiency because of the disease per se such as patients with 'Acquired Immune Deficiency Syndrome' (AIDS) or induced iatrogenically as a result of chemotherapy. Candida species are the most common cause of systemic fungal infections in the immuno-compromised patients(5) (Hawkins, 1984).

\section{Aims and Objectives}

1. To isolate, culture and sub-culture thereby identify the commonest subspecies of candida from the mucosal lesions of immuno-compromised individuals.

2. To study the age, sex distribution, dose and duration and the morphological pattern of lesions of immunosuppressed patients, predisposed to mucosal candidiasis.

\section{MATERIALS AND METHODS}

This descriptive study was conducted at the Government General Hospital, Chennai.

One hundred and twenty (120) patients who were either on immunosuppressive therapy or suffering from acquired immunodeficiency states, attending the Department of Dermatology were screened for the present study (Fig. No. 1). Study was carried out from $1^{\text {st }}$ July 2006 to $30^{\text {th }}$ September 2007. Cases were included in the study after confirming their diagnosis through appropriate investigations including histopathological studies and serological studies.

\section{Inclusion Criteria}

1. Patients should have cutaneous manifestations related to the disease with which they are suffering from.

2. Patients should have either been instituted therapy with systemic immunosuppressive drugs for the management of their disorders or should suffer from immunodeficiency because of the disease per se.

3. Development of candidiasis should be after the institution of immunosuppressive therapy.

\section{Exclusion Criteria}

1. Patients below 12 years of age.

2. Pregnant women.

3. Patients who have taken anti-fungal treatment (Topical/Systemic) within the past 6 months.

4. Patients with biological false positive serological tests.

Detailed case history of each patient was collected with reference to the duration of primary disease for which the immunosuppressive drug was given; appropriate note was also made regarding the dose and duration of each drug in their immunosuppressive regime.

Site, symptomatology and the duration of candidal infection that appeared after immunosuppressive therapy were recorded.

Detailed examination was also done to note down the other dermatological lesions.

\section{Mycological Examination}

Specimens such as mucosal scrapings were collected under aseptic precautions and examined microscopically in $10 \%$ potassium hydroxide $(\mathrm{KOH})$ solution for the presence of fungal elements.

Whenever the scraping was positive for budding yeast cells, hyphae or pseudohyphae (Fig. No. C-1), inoculation was done on Sabouraud's Dextrose Agar (SDA) (Media of pH 6.5) with chloramphenicol $(0.05 \mathrm{mg} / \mathrm{mL})$. Duplicate slants were maintained for all specimen.

All the inoculated slants were duly numbered and incubated at $37^{\circ} \mathrm{C}$ for a period of $24-48$ hours with everyday observation. Candida colonies appeared as white or cream coloured, smooth with a yeasty odour (Fig. No. C-2).

\section{SUB-CULTURE MEDIA $\rightarrow$ INDICATOR MEDIA HiCrome Candida Agar}

HiCrome candida agar is recommended for rapid isolation and identification of candida species from mixed cultures.

\begin{tabular}{|lc|}
\hline \multicolumn{1}{c}{ Composition } \\
Ingredients & Grams/ Litre \\
Peptic Digest of animal tissue & 15.0 \\
Agar & 15.0 \\
Chloramphenicol & 0.5 \\
Chromogenic mixture & 11.22 \\
Dipotassium hydrogen phosphate & 1.0 \\
Final pH (at $25^{\circ} \mathrm{C}$ ) & $6.3 \pm 0.2$ \\
\hline
\end{tabular}

\section{Constitution of Media}

21.36 gms of HiCrome candida agar is dissolved in $500 \mathrm{~mL}$ of distilled water. It is allowed to boil to dissolve the medium completely. Media should not be autoclaved. Media is then 
cooled to $50^{\circ} \mathrm{C}$ and aseptically poured into sterile petri plates (Fig. No. C-3).

\section{Sub-Culture}

Sub-culture of candida from SDA in HiCrome candida agar is done after 24 - 48 hours of primary culture.

\section{Principle and Interpretation}

Perry and Miller(6) reported that candida albicans produces an enzyme $\mathrm{B}-\mathrm{N}$-acetylgalactosaminidase and according to Rousselle et al,(7) incorporation of chromogenic or fluorogenic hexosaminidase substrates into the growth media helps in identification of candida albicans isolates directly on primary isolation. Hi-Crome candida agar media are selective and differential medium, which facilitates rapid isolation of yeasts from mixed cultures and allows differentiation on the basis of colouration and colony morphology.

Peptic digest of animal tissue, yeast extract, malt extract and glucose provides nitrogenous, carbonaceous, compounds and other essential growth nutrients. Chloramphenicol suppresses bacteria.

\begin{tabular}{|lcc|}
\hline \multicolumn{2}{c}{ Culture response } \\
Organisms & \multicolumn{1}{c|}{ Colour } \\
C.albicans & - & Light green (Fig No: C-4) \\
C.tropicalis & - & Light blue (Fig No: C-6) \\
C.krusei & - & White fuzzy (Fig No: C-5) \\
C.glabrata & - & Light pink (Fig No: C-4) \\
C.dubliniensis & - & Dark green (Fig No: C-6) \\
\hline
\end{tabular}

\section{RESULTS}

Out of 120 patients with immunosuppression subjected for the screening of candidiasis, 100 patients only were positive for candidiasis which includes 62 males and 38 females (Fig. No. 2). Their age ranged from 17 years to 85 years with a mean of 41.6 years.

\section{Age and Gender Distribution of Cases Positive for} Candidiasis

Both the patients in the extremes of age were males. The maximum number of Males (20) were in the age group of 31 40 years and maximum number of Females (13) were in the age group 21 - 30 years. The duration of their skin disease ranged from 3 weeks to 10 years.

Out of the 100 patients positive for candidiasis 60 patients suffered from various dermatological entities, among which Pemphigus vulgaris patients top the list (24) cases. The remaining 40 patients were victims of HIV.

Pemphigus vulgaris was the commonest bullous disorder that accounted for oral candidiasis (40\%).

Distribution of candidiasis in relation to various dermatoses on immunosuppressive therapy is as depicted in (Fig. No. 3)
Duration and Drug Regime of Immunosuppressive Therapy

- The duration of the therapy that predisposed to candidiasis ranged from 10 days to 1 year.

- Out of 100 patients, positive for candidiasis, 28 patients had taken treatment with Tab. Prednisolone $5 \mathrm{mg}$ alone taken in a dose range of $15 \mathrm{mg}$ to $20 \mathrm{mg}$ per day.

- 22 patients were treated with Inj. Dexamethasone (8 $\mathrm{mg}$ ).

- 5 patients were on Tab. Methotrexate $7.5 \mathrm{mg} /$ week.

- 2 patients were on combination of drugs viz.

- Inj. Dexamethasone (100 mg), and

- Inj. Cyclophosphamide (500 mg) (DCP) pulse therapy.

- Other combination of immunosuppressive drugs used were-

- T. prednisolone (15 mg) + Azathioprine (50 mg).

\section{Morphology of the Colonies}

After inoculation of the material under sterile aseptic precautions in SDA culture tubes with chloramphenicol, tubes were incubated at $37^{\circ} \mathrm{C}$. Creamy white, smooth individual colonies of candida with a yeasty odour were observed after 24 - 48 hours.

Individual colonies could be appreciated standing out as dome-shaped growth till 48 - 72 hours, since the time of inoculation after which the culture gets contaminated with saprophytic bacteria and other yeasts and moulds.

Sub-culture was made into HiCrome candida agar under sterile aseptic precautions and colonies were observed after 24 - 48 hours. Species of candida were identified with the help of the colour that they have produced by their interaction with the chromogenic indicator incorporated in the Hi-Media Crome Agar.

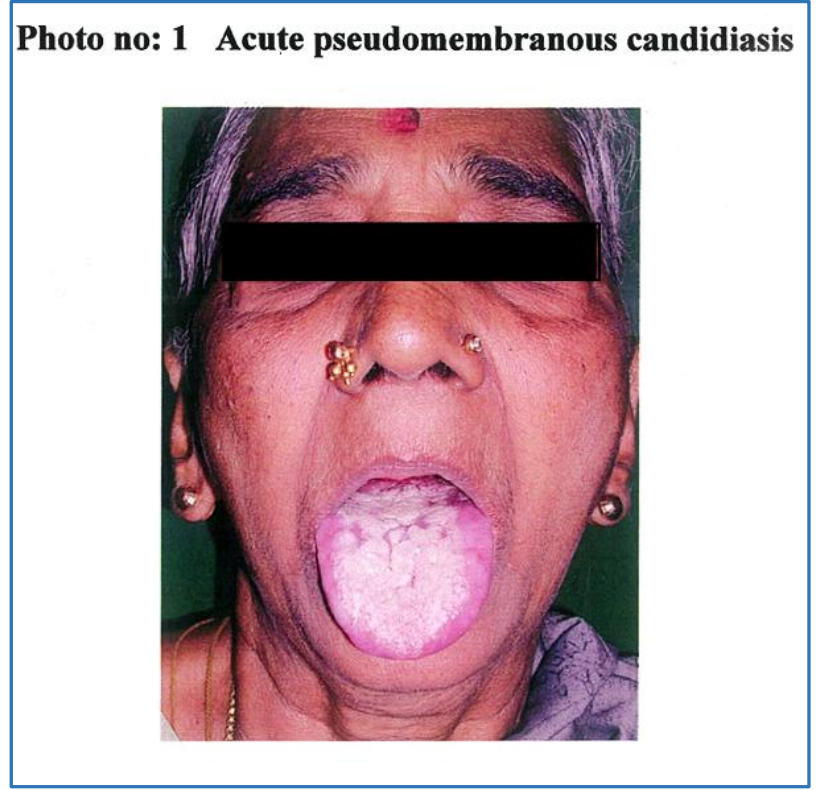




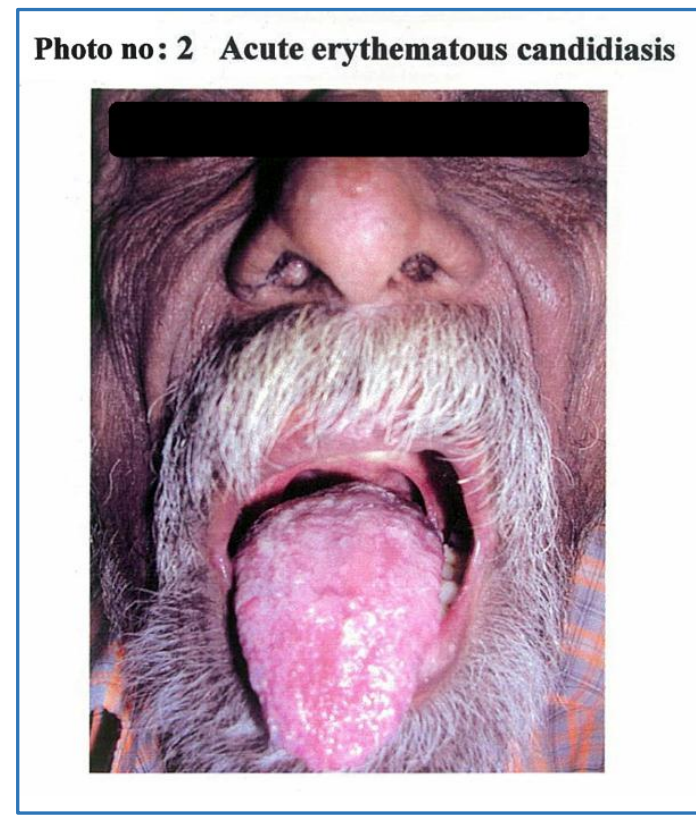

Photo no: 3

Angular cheilitis with Acute pseudomembranous candidiasis

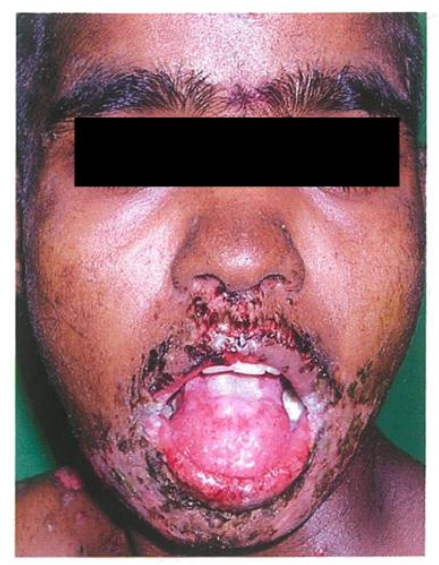

Photo no: 4 Median rhomboid glossitis

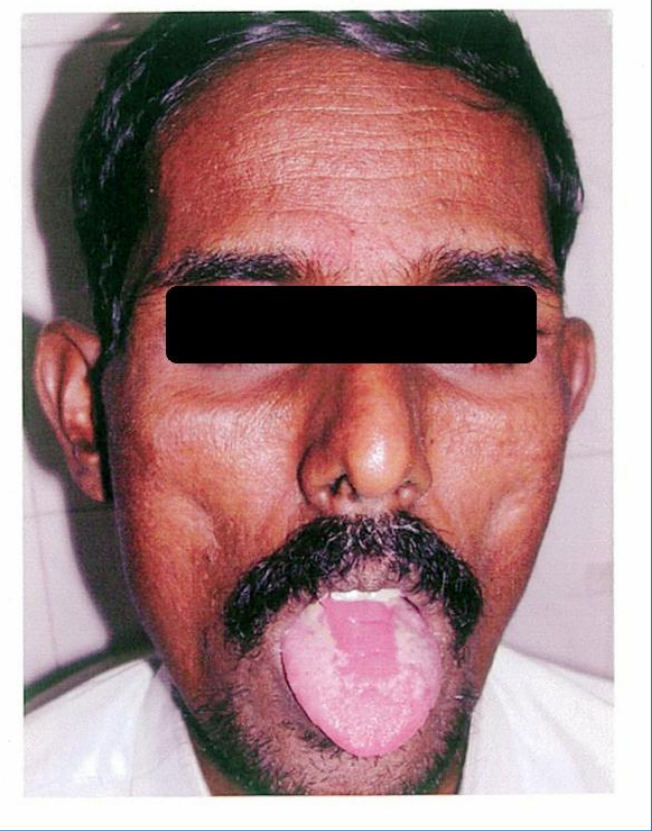

Photo no: 5

Chronic oral candidiasis with cerebriform fissuring of tongue

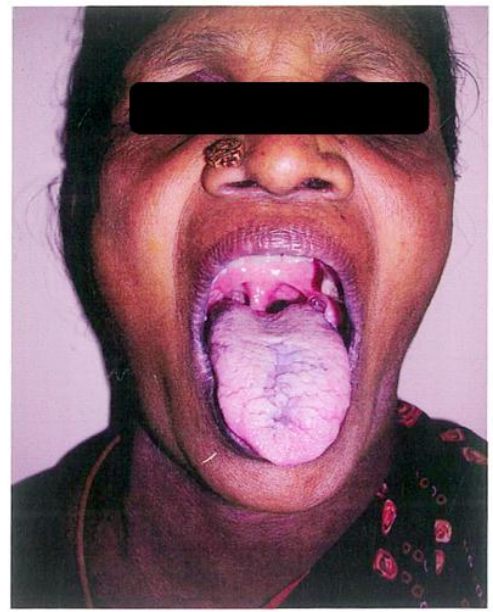

Photo no: 6

Oral candidiasis in HIV patient - showing deep midline fissuring of tongue

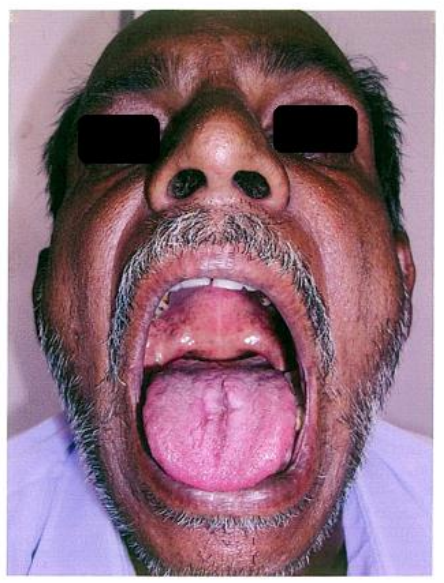

Photo no: 7

Oral candidiasis in HIV patient - showing candidial patches over hard and soft palate

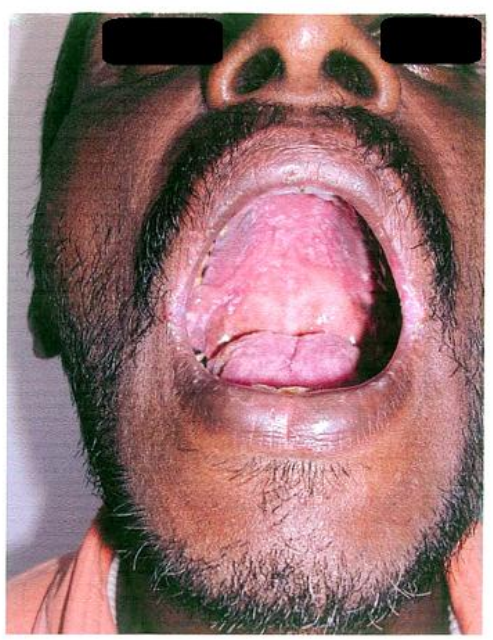




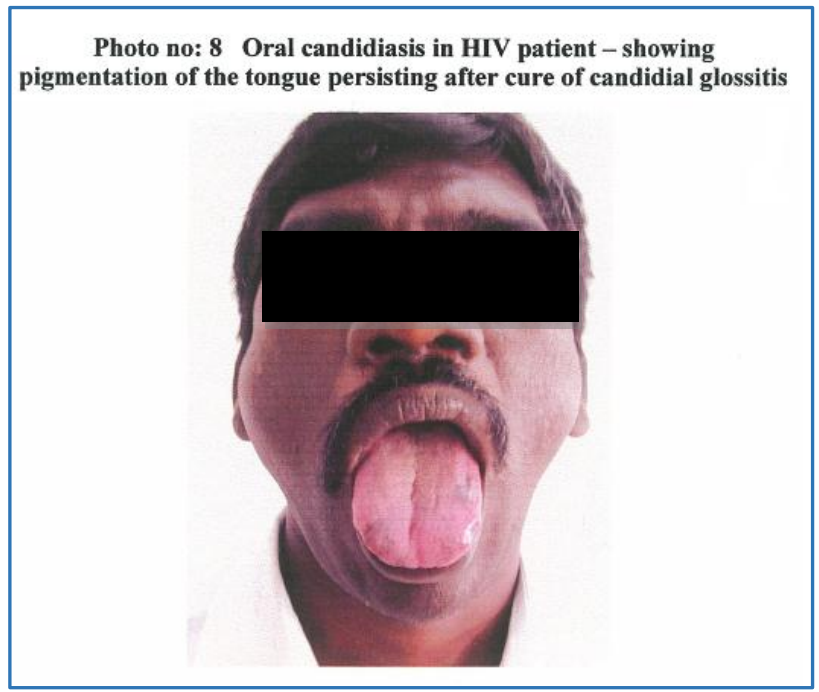

Photo no: 9 Candidial glossitis in HIV patient along with Herpes zoster of right supra and infra clavicular areas

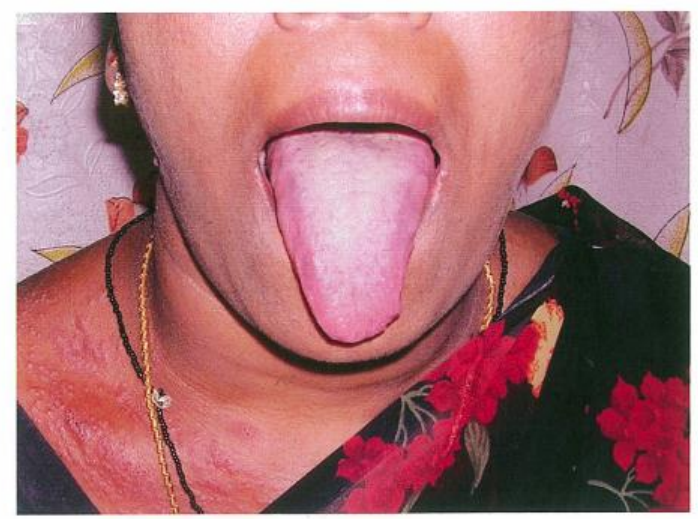

Photo no: 10

Candidial balano-posthitis in a diabetic individual

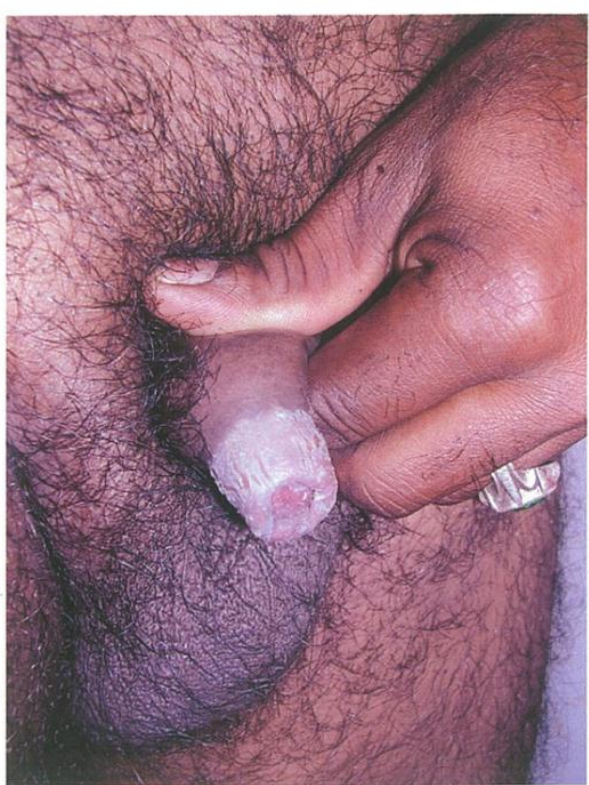

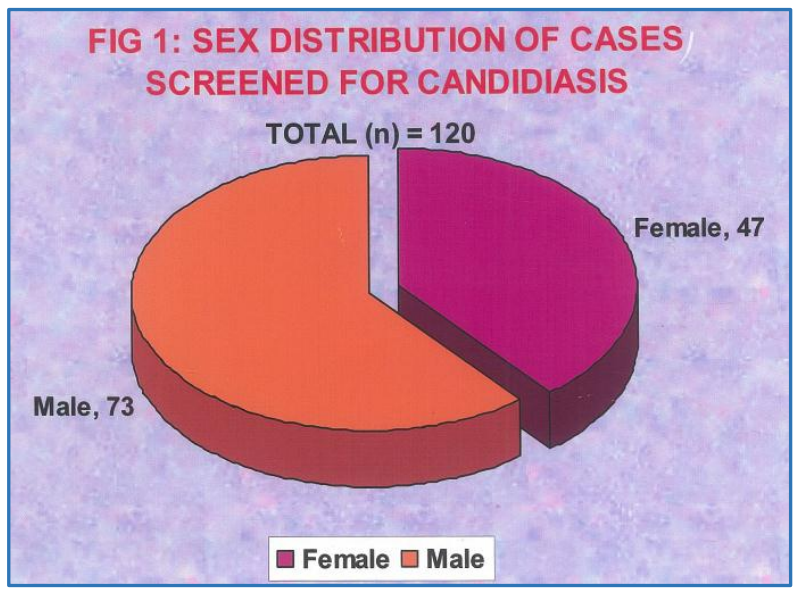
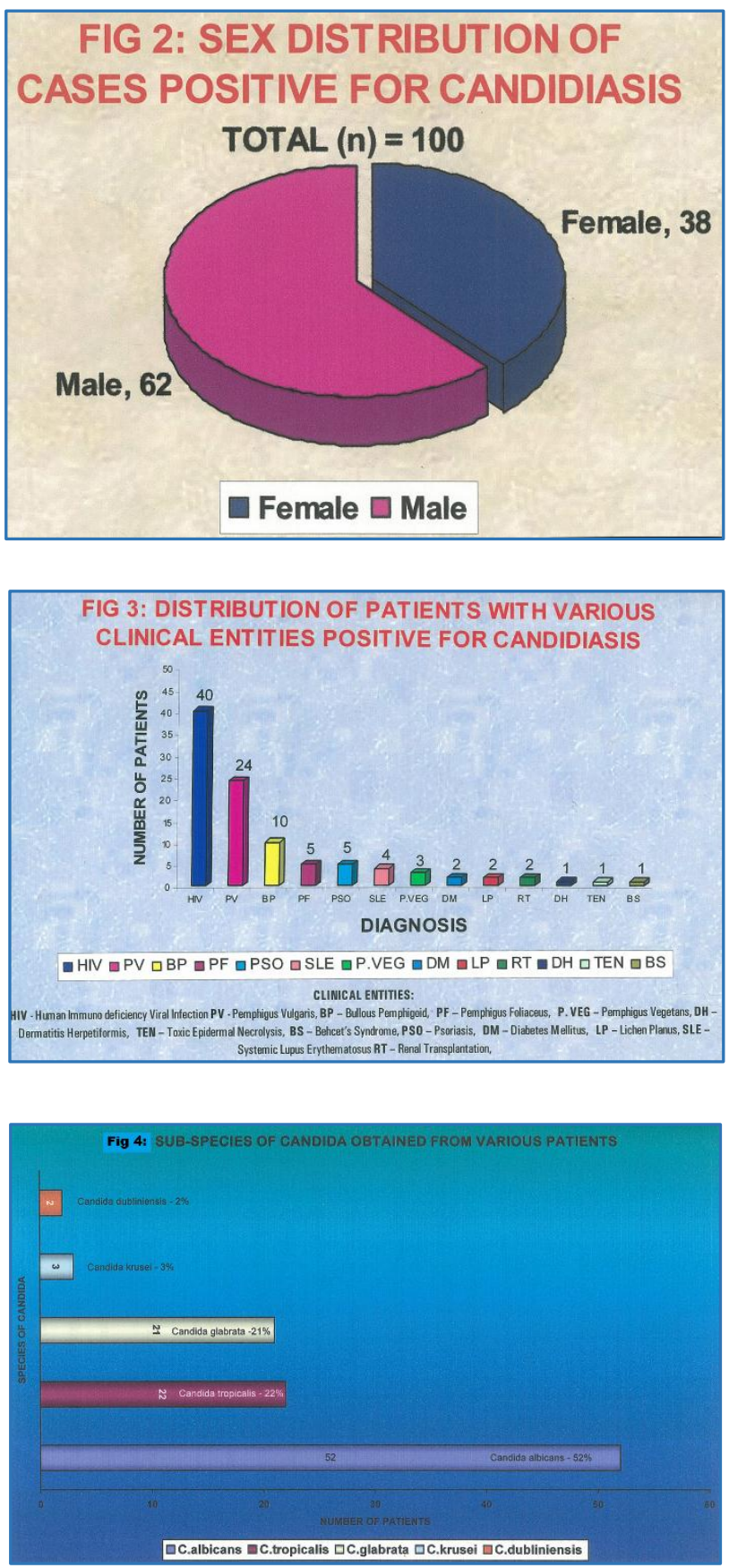

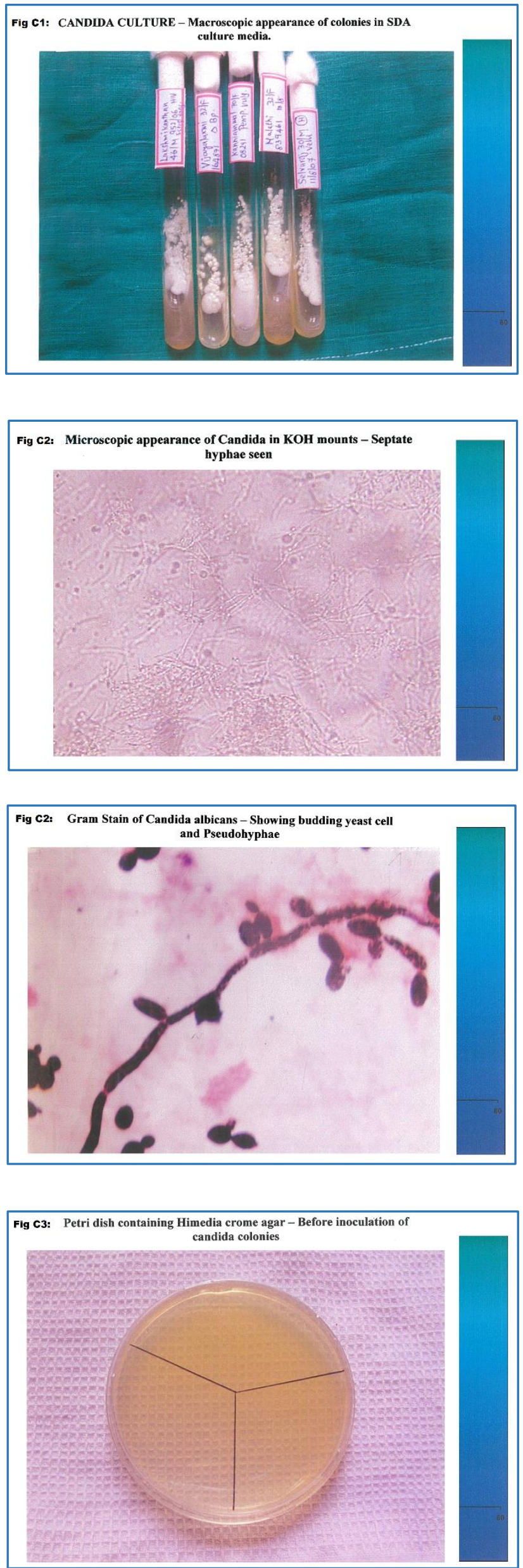
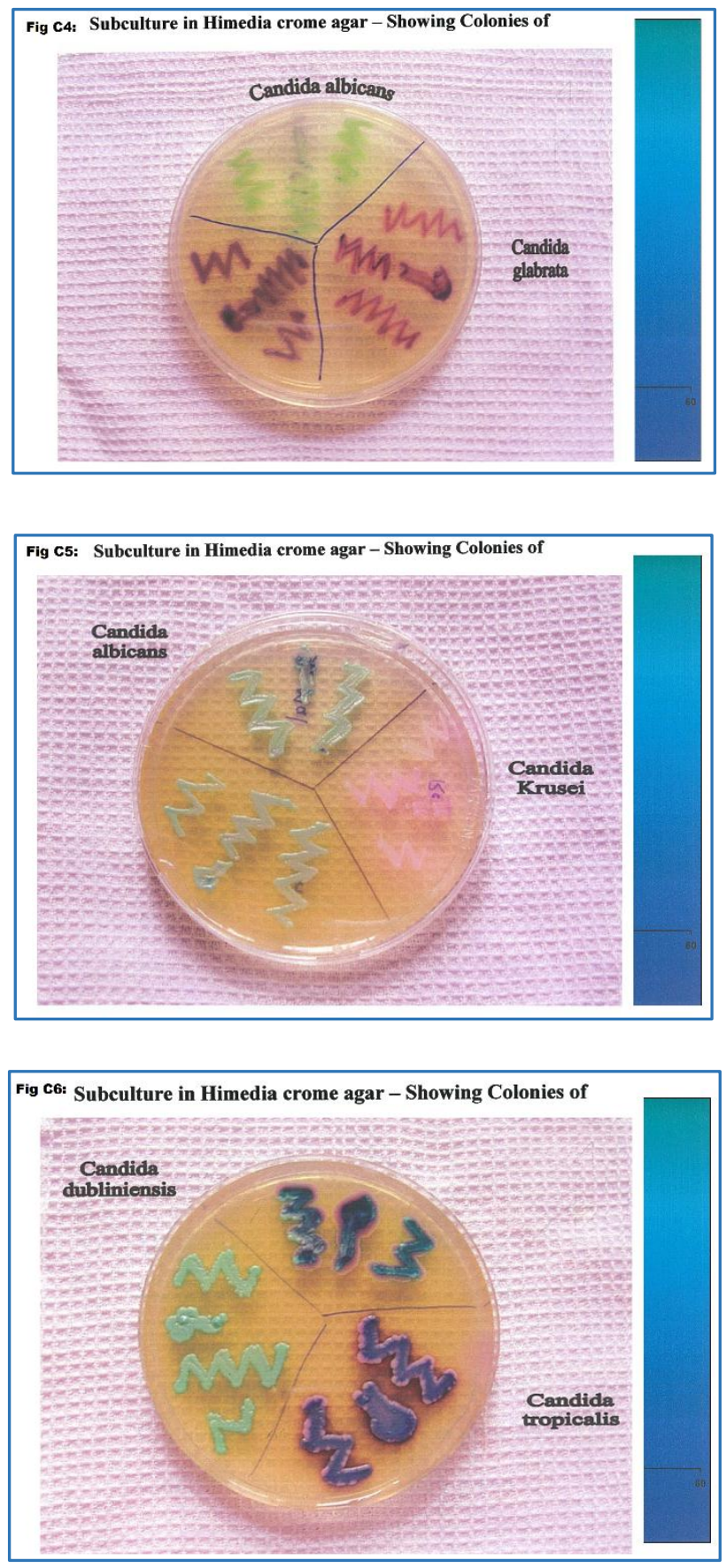

DISCUSSION

Appropriate statistical methods like calculation of mean, median and mode were enabled wherever applicable. In our present study, out of the 100 cases positive for candidiasis included majority of the patients were found to be in the age group of 31 - 50 years (53\%), out of which 35 were male patients and 18 were female patients. Patients in the extremes of age were males. The youngest male patient with oral candidiasis was a case of Bullous pemphigoid of age 17 years. The eldest patient was again a male of age 85 years, who was suffering from bullous pemphigoid.

Out of the 100 cases included in this study, 60 patients had various dermatoses and 40 cases were victims of HIV infection.

Of the 60 patients with various dermatoses studied, pemphigus vulgaris was the commonest clinical entity to be associated with oral candidiasis. 
24 cases of pemphigus vulgaris had oral candidiasis in our study (40\%). The next common bullous disorder to be associated with candidiasis was bullous pemphigoid. 10 cases of Bullous pemphigoid had oral candidiasis amounting to $16.6 \%$.

Incidence of oral candidiasis is more in Pemphigus vulgaris than Bullous pemphigoid because of the fact that nearly $70 \%$ of the cases of Pemphigus vulgaris have oral lesions, whereas only $30 \%$ of the Bullous pemphigoid cases present with oral lesions.

Other dermatoses to be associated with oral candidiasis in the decreasing order of frequency were- Pemphigus foliaceus (8.3\%), Psoriasis (8.3\%), Pemphigus vegetans (5\%), SLE (6.6\%), Lichen planus (3.3\%), Dermatitis herpetiformis (1.6\%), TEN (1.6\%) and Behcet's Syndrome (1.6\%).

Diabetes Mellitus was associated with candidiasis to an extent of 3.3\%.

2 male patients had diabetic balanoposthitis. Post renal transplant patients on immunosuppressive therapy were associated with oral candidiasis to an extent of $3.3 \%$.

\section{Immunosuppressive Drug}

In the present study the immunosuppressive drug commonly used was prednisolone, either alone or in combination with azathioprine or cyclophosphamide. The period of immunosuppressive therapy needed to produce candidiasis of the mucosal surfaces varies from a minimum of 10 days to maximum of 1 year. Combination of immunosuppressive drugs lead to persistence of candidal infection.

\section{Morphological Types of the Lesion}

In patients with bullous disorders who were on systemic steroids and other cytotoxic drugs like cyclophosphamide, azathioprine, etc. The commonest type of oral candidiasis encountered was acute pseudomembranous candidiasis (Thrush), characterised clinically by a sharply defined patch of creamy, crumbly, curd-like white pseudomembrane which when removed leaves an underlying erythematous base. The clinical changes were of the erosive type with severe symptoms resulting in inadequate food intake because of pain (Photo No. 1).

Acute erythematous candidiasis was the second commonest type of candidiasis that was encountered in our study. Marked soreness and denuded atrophic erythematous mucous membranes were noticed on the dorsal aspect of the tongue. This type might follow pseudomembranous candidiasis (Photo No. 2).

\section{Angular Cheilitis (Perleche)}

Soreness at the angles of the mouth extending outwards in the folds of the facial skin was noticed in all the patients of bullous dermatoses apart from their thrush lesions (Photo No. 3).

\section{Median Rhomboid Glossitis}

Median Rhomboid Glossitis type of candida lesion was encountered in one HIV infected male patient of age 40 years who was on antiretroviral therapy; condition was characterised clinically by a more or less diamond-shaped area on the dorsum of the tongue with loss of papillae(8) (Photo No. 4).
In cases of chronic oral candidiasis, deep fissuring of the tongue in a cerebriform pattern was noticed in a female patient suffering from pemphigus vulgaris (Photo No. 5).

In patients with HIV infection, deep longitudinal midline fissuring of the tongue was noticed (Photo No. 6).

Majority of the male patients with HIV infection with oral candidiasis had Addisonian type of oral pigmentation involving the hard and soft palates and also the buccal mucosa irrespective of their smoking habits. In HIV patients with $\mathrm{CD}_{4}$ count less than 100 cells/cu.mm, candidal patches were also noticed in the hard and soft palate apart from the lesions over the tongue (Photo No. 7).

The black pigmentation persisted even after the clinical cure of the lesions (Photo No. 8).

The female patient with HIV infection had multidermatomal herpes zoster as an associated dermatological entity (Photo No. 9).

In Diabetic individuals candidal balanoposthitis was associated with soddening and linear fissuring; prepuce was oedematous and there was sub-preputial discharge because of the associated phimosis $\left({ }^{(9)}\right.$ (Photo No. 10).

The following species of candida were isolated on doing sub-culture in Hi-Media Crome Agar(10) viz. Candida albicans (52\%), C. tropicalis (22\%), C. glabrata (21\%), C. krusei (3\%) and C. dubliniensis (2\%) (Fig. No. 4). Candida dubliniensis was isolated from the oral lesions of two HIV positive patients. C. dubliniensis produces dark green colour in $\mathrm{Hi}-$ Media Crome Agar (Photo No. 11).

In the female patient with $\mathrm{C}$. dubliniensis infection, the $\mathrm{CD}_{4}$ count was less than 50 cells/ cu.mm and the patient had verruca vulgaris as an associated opportunistic infection. Acute pseudomembranous glossitis was the clinical type of lesion in this patient.

The commonest systemic disease associated with HIV patients with oral candidiasis was pulmonary tuberculosis.

\section{CONCLUSION}

- Candidiasis is the commonest mycosis in patients on systemic immunosuppressive therapy and immunosuppressive status like HIV infection.

- Majority of the affected victims of candidiasis were in the age group between 31 - 50 years. Incidence of candidiasis was found to be more in male patients than female patients. (Males $\rightarrow 62 \%$ ), (Females $\rightarrow 38 \%$ ) (Fig. No. 2). However, the age and sex distribution is also related to the patients screened.

- Acute pseudomembranous glossitis was the commonest morphological type of the lesion encountered in this study (35\%) followed by chronic erythematous candidiasis (22\%), Angular cheilitis (21\%), acute erythematous glossitis (19\%), balanoposthitis (2\%) and median rhomboid glossitis (1\%).

- Candida albicans was the commonest species isolated (52\%). Other species isolated include Candida tropicalis (22\%), Candida glabrata (21\%), Candida krusei (3\%) and Candida dubliniensis (2\%).

- $\quad$ Even in HIV infected individuals candida albicans was the commonest species isolated in our study; candida dubliniensis is said to be exclusively common in the oral lesions of HIV patients, which however accounted only for $(2 \%)$ in our study. Probably, the sample size had still been larger. 
- Among the various immunosuppressive drugs taken into account for this study, Tab. Prednisolone was the commonest drug predisposing to candidiasis (28\%) followed by Inj. Dexamethasone (22\%). Thus, systemic steroids accounted for $50 \%$ of drugs predisposing to candidosis, remaining $50 \%$ being contributed by cytotoxic drugs like methotrexate, cyclophosphamide, azathioprine, etc.

- Of all the various Bullous dermatoses screened, Pemphigus vulgaris was the commonest bullous disorder predisposing to candidosis (40\%) followed by Bullous pemphigoid (16.6\%), Pemphigus foliaceus (8.3\%), Dermatitis Herpetiformis (1.6\%) and Pemphigus vegetans (5\%).

- The minimum time duration of systemic immunosuppressive therapy required for predisposition to oral candidosis was found to be 10 (Ten) days in our study in Non-HIV infected individuals, which was found to be still shorter in known cases of HIV infection (around 1 week); probably due to $\mathrm{CD}_{4}$ cell count depletion and other associated opportunistic infections.

- Patients with $0+v e$ blood group are considered to have high carriage of candida species.

- In spite of heroic measures to avoid contaminants and adhering to very strict aseptic protocols, it was found in our study that the maximum keeping time of primary candida culture in SDA with chloramphenicol and subcultures in Hi-Media Crome Agar was just 72 hours, after which saprophytic bacteria and various moulds and concomitant pathogens spoil the culture media.

\section{REFERENCES}

[1] Chander J. A textbook of medical mycology. 22 $2^{\text {nd }}$ edn. 1996:131-2.

[2] McCourtie J, Douglas LJ. Relationship between cell surface composition, adherence and virulence of candida albicans. Infect Immun 1984;45(1):6-12.

[3] Kaufman R. Vulvovaginal candidiasis: a symposium. J Reprod Med 1986;31(7):639-72.

[4] Douglas LJ. Adhesion to surfaces. In: Rose AH, Harrison JS. (eds). The yeasts. Vol 2. London: Academic Press, 1987:239-80.

[5] Hawkins C, Amstrong D. Fungal infection in the immunecompromised host. Clin Haematol 1984;13(3):599-630.

[6] Perry JL, Miller GR. Umbelliferyl-labeled galactosaminide as an aid in identification of Candida albicans. J Clin Microbiol 1987;25(12):2424-5.

[7] Rousselle P, Freydiere AM, Couillerot PJ, et al. Rapid identification of Candida albicans by using Albicans ID and fluoroplate agar plates. J Clin Microbiol 1994;32(12):3034-6.

[8] Cooke BE. Median rhomboid glossitis. Candidiasis and not a developmental anomaly. $\mathrm{Br} \mathrm{J}$ Dermatol 1975;93(4):399-405.

[9] Odds FC. Genital candidiasis. Clin Exp Dermatol 1982;7:343-54.

[10] Odds FC, Bernaerts R. CHROMagar candida, a new differential isolation medium for presumptive identification of clinically important candida species. J Clin Microbiol 1994;32(8):1923-9. 